\title{
Benchmarking to Assess Potential Under-Diagnosis of Smear-Negative and Extrapulmonary Tuberculosis. A Case Study from Mozambique
}

\author{
Miranda Brouwer, ${ }^{*}$, Paula Samo Gudo ${ }^{2}$, Chalice Mage Simbe ${ }^{3}$, Paula Perdigão ${ }^{4}$ and \\ Frank van Leth ${ }^{5,6}$ \\ ${ }^{1}$ Health Alliance International, Technical Assistance Unit, Maputo, Mozambique \\ ${ }^{2}$ Ministry of Health, National TB Programme, Mozambique \\ ${ }^{3}$ Ministry of Health, Provincial Health Directorate Manica Province, Chimoio, Mozambique \\ ${ }^{4}$ Phoenix Centro Medico, Maputo, Mozambique \\ ${ }^{5}$ Department of Global Health, Academic Medical Center, University of Amsterdam, Amsterdam Institute for Global \\ Health and Development, The Netherlands
}

${ }^{6}$ KNCV Tuberculosis Foundation, The Hague, The Netherlands

\begin{abstract}
No tool exists to identify potential under-diagnosis of smear-negative and extrapulmonary TB. In an ecological study, we used a simple tool plotting percentages of smear-positive pulmonary TB amongst newly diagnosed TB in African region countries and provinces in Mozambique against the country's and provinces' HIV prevalence. We visually inspected the plots to determine potential under-diagnosis of smear-negative and extrapulmonary TB in three facilities in Manica Province, Mozambique.

In these facilities, we found $67 \%$ smear-positivity among new TB cases in an area with HIV prevalence of more than $10 \%$. All African region countries with an HIV prevalence of more than $10 \%$ had a smear-positivity lower than $50 \%$. Most Mozambican provinces with a high HIV prevalence have smear-positivity rate of below 51\%. Our findings show that benchmarking can be used to assist in identifying potential under-diagnosis of smear-negative and extrapulmonary TB. The findings also suggest that potentially under-diagnosis of these forms of TB exists in the study population.
\end{abstract}

Keywords: Mozambique, routine programme data, tuberculosis, HIV, under-diagnosis, benchmarking.

\section{INTRODUCTION}

Tuberculosis (TB) is a serious health problem in Mozambique with estimated incidences of over 500/100.000 for all forms. Co-infection with Human Immunodeficiency Virus (HIV) is high: $66 \%$ of the tested TB patients [1].

In Mozambique, like in many countries, the diagnosis of TB is mainly based on sputum smear microscopy, which assesses the presence of acid-fast bacilli. When present, the patient is registered as 'smear-positive' and started on TB treatment. Smear-negative TB refers to those patients who have sputum that is negative on smear microscopy. Another category of patients is of those who have TB outside the lungs (extrapulmonary TB). In HIV-infected individuals smear-negative and extrapulmonary $\mathrm{TB}$ is more frequent than in HIV-negative individuals [2]. In addition, patients with smear-negative pulmonary $\mathrm{TB}$ have worse treatment outcomes than those with smear-positive pulmonary TB [3]. This is partly because patients with smear-negative pulmonary $\mathrm{TB}$ are more often HIV-infected. Furthermore,

*Address correspondence to this author at the Health Alliance International, Technical Assistance Unit, Maputo, Mozambique; Tel: 00258826007836; E-mail: brouwer.miranda@gmail.com mortality in HIV-infected TB patients is higher than in those not HIV-infected [2].

Diagnosis of smear-negative and extrapulmonary TB is a major challenge in settings where smear microscopy is the main TB diagnostic test. The World Health Organization (WHO) recommends the use of chest radiography in TB suspects with negative smear microscopy results, however, this is not available everywhere [4]. In the absence of other diagnostic tools, the diagnosis of smear-negative and extrapulmonary TB is often based on clinical grounds. But clinicians often are reluctant to treat smear-negative TB because they lack certainty of the diagnosis [5]. This may lead to under-diagnosis of smear-negative and extrapulmonary TB, especially in the HIV-infected patients, and withholding of a potential life-saving treatment to a large group of patients.

The diagnostic challenge of smear-negative and extrapulmonary $\mathrm{TB}$ is reflected in a high percentage of smear-positive TB observed during routine supervision visits in Manica province, Mozambique, our study area. We found this high percentage of smear-positive TB remarkable because one would have expected more smear-negative and extrapulmonary TB in this high HIV prevalence area. We considered the possibility that smear-negative and 
extrapulmonary TB were under-diagnosed. However, currently we lack a tool to assess potential under-diagnosis of smear-negative and extrapulmonary TB using routine data of a national TB programme. Comparison of individual clinicians' diagnostic processes is cumbersome. This is further complicated by the fact that one does not know what the expected percentage of non smear-positive TB in a specific setting or region should be. Nonetheless, if TB programme staff and management could use routinely available data to assess potential under-diagnosis of smearnegative and extrapulmonary $\mathrm{TB}$, it would be easier to undertake action.

Benchmarking with routinely available data would provide a method to assess the TB diagnostic process. Therefore, we addressed in this ecological study the following research question. Can routinely reported notification data of other countries and provinces expressed as the percentage of new smear-positive TB amongst new TB cases serve as a benchmark in identifying potential under-diagnosis of smear-negative and extrapulmonary TB in our setting?

\section{METHODS}

In a retrospective ecological study, we included all new TB cases of patients of 15 years and older notified from $1^{\text {st }}$ January to $31^{\text {st }}$ December 2007 in three health facilities in Manica Province, Mozambique. In 2007, the participating facilities notified 48 children with TB. These were excluded because the TB diagnostic process in children is more complicated than in adults. This exclusion may affect the positivity rate as children usually have smear-negative and extrapulmonary disease [6].

Manica province is situated in the centre of Mozambique and had in 2007 a population of 1.4 million people. The province notified 1,529 new TB cases in 2007. The three health facilities were selected on the presence of both TB and HIV treatment services in the facility and at least 150 TB patients notified in 2007. The three facilities together notified almost $40 \%$ of the provincial new TB cases in that year. We collected the following data from the TB registers of the health facilities: age, sex, type of TB, sputum smear microscopy results at diagnosis and HIV test results.

We compared the TB notification data from the study population with aggregated TB notification data from two different sources. The first source was the WHO's global TB report which is available in the public domain. From this we extracted the percentage of smear-positive TB among all new notified TB in 2007 for all countries in the African region [1]. We used the African region countries because their diagnostic process is similar to the one in Mozambique. In addition we collected the HIV prevalence for the African region countries from UNAIDS [7].

The second source was the 2007 notification data in the 11 provinces of Mozambique from the National TB Programme. From these we extracted the percentage of smear-positive TB among all new notified TB. A population study from Mozambique provided the HIV prevalence data for each of the 11 provinces [8].

We plotted the percentage of smear-positive TB among all new notified TB against the HIV prevalence in the African region country or the Mozambican province. We assessed potential under-diagnosis by visual inspection of these plots.

The National Bio-ethic Committee of the Mozambican Ministry of Health approved the protocol. For Health Alliance International, the Institutional Review Board of the University of Washington in Seattle, USA approved the protocol. We did not obtain informed consent because we used routine data.

\section{RESULTS}

The three health facilities recorded in total 591 new patients of 15 years and older in 2007. Of these, $67 \%$ had smear-positive TB. The patient characteristics by type of TB of the study population are summarized in Table 1 . The estimated HIV prevalence for the study area is $15.3 \%$ and for Mozambique as a whole $11.5 \%$ [8].

Health facility $A$ in the provincial capital had $48 \%$ smear-positive TB and health facilities B and C, respectively 20 and 80 kilometres away from the provincial capital had $78 \%$ and $67 \%$ smear-positive TB. Among the tested TB patients, the HIV-positivity rate was $70 \%$ in facility $\mathrm{C}, 71 \%$ in facilities A and B.

We obtained information on TB notification and HIV prevalence for 42 countries in the African region. Of the countries with HIV prevalence of $10 \%$ or below, $81 \%$ had a percentage of smear-positive TB greater than 50\%. All countries with an HIV prevalence of more than $10 \%$ had a percentage smear-positive TB equal or less than $50 \%$ (Fig. 1). With an estimated HIV-prevalence in the study area of $15.3 \%$ and $67 \%$ of the newly registered patients classified

Table 1. Patient Characteristics of the Study Population (Three Health Facilities in Manica Province) According to Type of Tuberculosis

\begin{tabular}{|c|c|c|c|c|c|c|}
\hline & \multicolumn{2}{|c|}{$\begin{array}{c}\text { Smear-Positive Tuberculosis } \\
(\mathbf{n}=395)\end{array}$} & \multicolumn{2}{|c|}{$\begin{array}{l}\text { Smear-Negative and Extrapulmonary } \\
\text { Tuberculosis }(\mathrm{n}=196)\end{array}$} & \multicolumn{2}{|c|}{ Total $(n=591)$} \\
\hline & Number & $\%(95 \%$ CI $)$ & Number & $\%$ & Number & $\%$ \\
\hline Female sex & 182 & 46 & 93 & 48 & 275 & 47 \\
\hline Age, median $\left(\mathrm{IQR}^{*}\right)$ in years & $30(24-40)$ & & $35(27-42.8)$ & & $32(25-41)$ & \\
\hline Tested for HIV & 311 & 79 & 167 & 85 & 478 & 81 \\
\hline
\end{tabular}




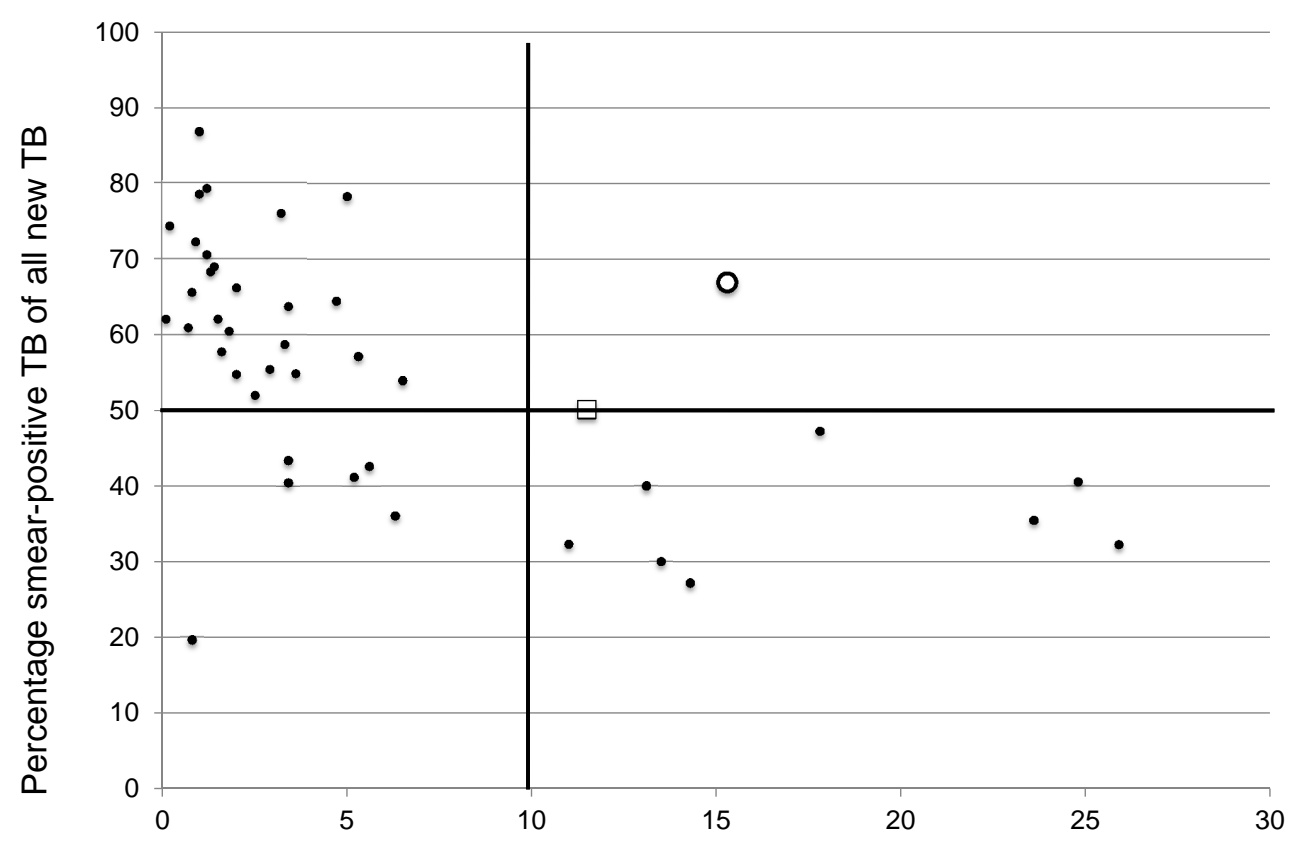

HIV prevalence in the general population (in \%)

Legend: • African region country, $\bigcirc$ study population, $\square$ Mozambique

Fig. (1). HIV prevalence and smear-positivity rate among new TB cases notified in 42 African region countries and the study population.

as smear-positive, the data from the study population do not fit with routine data from other countries.

Fig. (2) shows this approach for the 11 provinces of Mozambique. It shows the plot of the 2007 notification data of all eleven Mozambican provinces (Table 2). All provinces with an HIV prevalence of more than $10 \%$ had a percentage of smear-positive TB of $51 \%$ or below apart from Manica province. This province had a smear-positivity rate of $67 \%$, the same as we found in our study population that forms part of Manica province. The HIV prevalence was $15.3 \%$ in Manica, including the study population.

In both the comparison with African region countries and with other Mozambican provinces the data of the study population form an outlier.

\section{DISCUSSION}

The results of our study show that the percentage of smear-positive TB among newly registered cases in the study

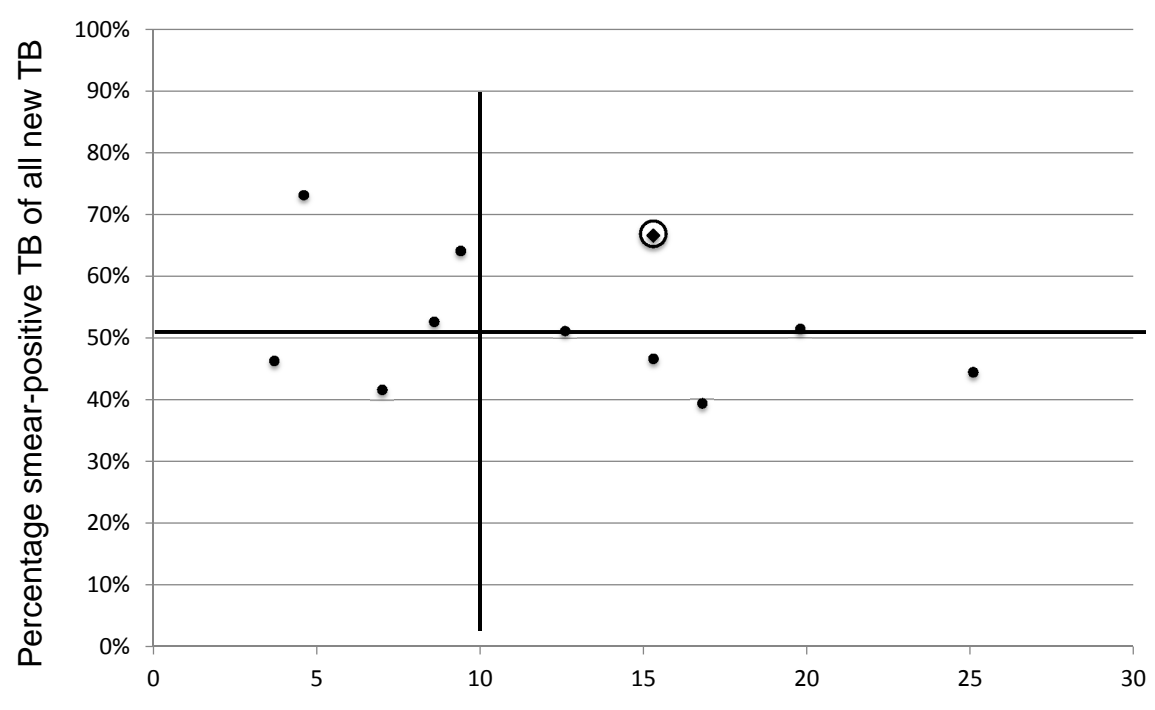

HIV prevalence in the general population (in \%)

Legend: • Mozambican province, $\mathbf{O}$ study population

Fig. (2). HIV prevalence and smear-positivity rate among new TB cases notified in 11 Mozambican provinces and the study population. 
Table 2. TB Notification Data for 2007 for Eleven Mozambican Provinces

\begin{tabular}{|c|c|c|c|c|c|}
\hline Niassa & 388 & 326 & 93 & $48 \%$ & 3.7 \\
\hline Cabo Delgado & 1,221 & 807 & 158 & $56 \%$ & 9.4 \\
\hline Nampula & 3,006 & 1,013 & 270 & $70 \%$ & 4.6 \\
\hline Tete & 970 & 1,313 & 369 & $37 \%$ & 7.0 \\
\hline Manica & 1,235 & 605 & 218 & $60 \%$ & 15.3 \\
\hline Sofala & 2,858 & 2,147 & 651 & $51 \%$ & 15.3 \\
\hline Inhambane & 1,030 & 1,132 & 279 & $42 \%$ & 8.6 \\
\hline Study population & 395 & 163 & 33 & $67 \%$ & 15.3 \\
\hline
\end{tabular}

area is markedly different from that seen in other countries from the African region and in other provinces in Mozambique with a similar HIV prevalence. Only Manica province, of which the study population is a part, had the same smear-positivity rate as the study population. This analysis provides an indication of potential under-diagnosis of smear-negative and extrapulmonary $\mathrm{TB}$ in the study population. In this way the use of routine data on the percentage of smear-positive TB of all new TB cases from other countries or other areas in the country can serve as a benchmark in identifying potential under-diagnosis of smearnegative and extrapulmonary TB.

Given the readily availability of these data through the annual WHO TB report, or the routinely collected data within an NTP, the study introduces a simple tool for benchmarking the performance of the own TB programme or specific provinces within a single country. This benchmarking can assist with the identification of areas in the TB programme that show different programme outcome than expected when compared to similar areas. Because of the absence of the 'correct percentage of smear-positivity amongst new TB patients', the identification of an outlier when benchmarking, is by no means proof of an abnormal situation. It should however, lead to an investigative action into the reasons for the encountered difference.

The results show that especially in high HIV prevalence areas the clinicians do not solely rely on the results of smear microscopy to initiate TB treatment. This clinical judgment is appropriate where other diagnostic tools are scarce.

Identifying the study area as having potential underdiagnosis of smear-negative and extrapulmonary TB is not surprising as the main diagnostic tool is smear microscopy in most of the area. Outside the provincial capital, radiology services are scarce and not always operational. Chest X-ray plays a crucial role in the diagnosis of smear-negative TB [4], the absence of which seems to affect Manica province, to which our study population belongs as well, more severe than other provinces in Mozambique. We did not evaluate whether chest X-ray was used in the diagnosis of smearnegative TB. However, the difference between the individual facilities showed a high percentage of smear-negative and extrapulmonary TB in the provincial capital where chest Xray is relatively close available.

Diagnosis of smear-negative and extrapulmonary TB is recognized as a challenge, especially in countries with a high HIV prevalence [9]. A study in Malawi, a neighbor of Mozambique with a similar HIV prevalence, showed that with a reasonable adherence to diagnostic guidelines, diagnosis of smear-negative TB is possible [10]. A smearpositivity of $32 \%$ amongst newly registered patients in Malawi reflects this adherence to the diagnostic guidelines.

Even following the guidelines will leave some TB cases undiagnosed. Wood and colleagues found a substantial number of undiagnosed cases in the community, half of which were smear-negative [11]. The majority of these cases were in HIV-infected individuals. Fortunately, a new diagnostic tool has become available recently. This automated molecular test for TB shows $72 \%$ sensitivity in smear-negative, culture positive pulmonary TB [12]. However, the cost of this test is a major limitation in resource-limited settings. A much cheaper urine dipstick test shows great promise, especially in HIV-infected patients with advanced immunosuppression [13]. Though not yet available, it would be very useful in settings like Manica province where HIV prevalence is high.

\section{LIMITATIONS}

A limitation of our study was that other countries may use a different diagnostic process and this affects the smearpositivity percentage. Including a large number of countries in the benchmarks most likely evens-out these effects in different directions. Furthermore, to our knowledge most African region countries did have limited access to culture at the time of the study and thereby would have similar 
diagnostic processes based mainly on smear microscopy, as is the case in Mozambique.

We did not look at individual patient records and were therefore not able to assess the patient-by-patient diagnostic procedure of non smear-positive TB. This would have given us more information on the diagnostic procedure; however, our purpose was to work with easy and readily available data. The fact the patient was registered as a smear-negative TB case indicates that there was sufficient clinical suspicion to start TB treatment.

A further limitation is that we compared notifications in adults only with country and province data that also include children. Smear-negative and extrapulmonary TB is more common in children and we could have overestimated the smear-positivity rate in our study population [6]. However, if we consider all these children to be smear-negative or extrapulmonary $\mathrm{TB}$, the smear-positivity rate would have been $62 \%$ and still much higher than the $50 \%$ observed in other high HIV prevalent settings.

This benchmark is not a commonly used tool in TB control. However, comparing the performance of one country against other countries is quite common, for example in the Global TB Report published annually by WHO [1]. The comparison is not proof of underdiagnosis of smear-negative or extrapulmonary TB. Notification data of non smear-positive TB depend on various factors including access to quality smear microscopy, available resources to start treatment in suspects with no bacteriological confirmation of the diagnosis, and others. However, the main use of the proposed benchmarking is to have a tool that can alert programme management to a difference in programme performance compared to similar settings that merits further analysis.

\section{CONCLUSION}

We conclude that notification data of other countries and of provinces in the same country can serve as a benchmark to assist identifying potential under-diagnosis of smearnegative and extrapulmonary TB. This benchmark tool can assist countries and sub national levels to quickly and easily assess if their TB diagnosis is comparable to similar settings.

Identifying potential under-diagnosis of smear-negative and extrapulmonary TB through benchmarking is not a purpose in itself. The aim is to improve diagnosis of these forms of TB, which is challenging but possible with existing tools. Further research could focus on whether using the benchmark tool leads to interventions that improve diagnosis of non smear-positive TB.

\section{CONFLICT OF INTEREST}

The authors confirm that this article content has no conflicts of interest.

\section{ACKNOWLEDGEMENTS}

The authors thank the data collectors Celina Joaquim, Eugénio Alberto Anselmo, and Nelson Chico Mafione. We thank Pedro Tenente for support with the data collection. The authors also wish to acknowledge the support of the National Tuberculosis Programme within the Mozambique Ministry of Health.

\section{REFERENCES}

[1] World Health Organization. Global Tuberculosis Control 2010 Annual Report WHO/HTM/TB/2010.7. Switzerland: WHO 2010.

[2] Corbett EL, Marston B, Churchyard GJ, De Cock KM. Tuberculosis in sub-Saharan Africa: opportunities, challenges, and change in the era of antiretroviral treatment. Lancet 2006; 367 : 926-37.

[3] Harries AD, Nyirenda TE, Banerjee A, Boeree MJ, Salaniponi FM. Treatment outcome of patients with smear-negative and smearpositive pulmonary tuberculosis in the National Tuberculosis Control Programme, Malawi. Trans R Soc Trop Med Hyg 1999; 93: 443-6.

[4] World Health Organization. Improving the diagnosis and treatment of smear-negative pulmonary and Extrapulmonary tuberculosis among adults and adolescents. Recommendations for HIVprevalent and resource-constrained settings. WHO /HTM /TB /2007.379. Switzerland: WHO Press 2007.

[5] Basinga P, Moreira J, Bisoffi Z, Bisig B, Van den Ende J. Why are clinicians reluctant to treat smear-negative tuberculosis? An inquiry about treatment thresholds in Rwanda. Med Decis Making 2007; 27: 53-60.

[6] Nelson LJ, Wells CD. Global epidemiology of childhood tuberculosis. Int J Tuberc Lung Dis 2004; 8: 636-47.

[7] UNAIDS. UNAIDS Report on the Global AIDS Epidemic 2010. Global Report. UNAIDS/10.11E | JC1958E. US: UN Joint Programme on HIV/AIDS 2010.

[8] Ministério de Saúde 2009. Inquérito nacional de prevalência, riscos comportamentais e informação sobre o HIV e SIDA emMoçambique (INSIDA 2009) relatório final, 2009.

[9] Colebunders R, Bastian I. A review of the diagnosis and treatment of smear-negative pulmonary tuberculosis. Int $\mathbf{J}$ Tuberc Lung Dis 2000; 4: 97-107.

[10] Harries AD, Hargreaves NJ, Kwanjana JH, Salaniponi FM. Clinical diagnosis of smear-negative pulmonary tuberculosis: an audit of diagnostic practice in hospitals in Malawi. Int J Tuberc Lung Dis 2001; 5: 1143-7.

[11] Wood R, Middelkoop K, Myer L, et al. Undiagnosed Tuberculosis in a Community with High HIV Prevalence. Implications for Tuberculosis Control. Am J Respir Crit Care Med 2007; 175: 8793.

[12] Boehme CC, Nabeta P, Hillemann D, et al. Rapid molecular detection of tuberculosis and rifampin resistance. N Engl J Med 2010; 363: 1005-15.

[13] Lawn SD, Kerkhoff AD, Vogt M, Wood R. Diagnostic accuracy of a low-cost, urine antigen, point-of-care screening assay for HIVassociated pulmonary tuberculosis before antiretroviral therapy: a descriptive study. Lancet Infect Dis 2012; 12: 201-9. 